\title{
Exodus und Rückkehr der Rechtssoziologie
}

Die Rechtssoziologie hat ihre Konjunkturen. Eine erste Blüte erlebte die Disziplin vor dem ersten Weltkrieg. Eugen Ehrlich wendete sich dem in der Gesellschaft „lebenden Recht“" zu. Der Rechtsanwalt Hugo Sinzheimer referierte 1909 vor dem Sozialwissenschaftlichen Verein der Universität München über die Anwendung der soziologischen Methode in der Privatrechtswissenschaft. Der erste Soziologentag 1910 diskutierte Beiträge über rechtsbezogene Themen, u.a. den von Hermann Kantorowicz über „Rechtswissenschaft und Soziologie“. ${ }^{1}$ Der Deutsche Juristentag befasste sich 1912 mit der Frage ,Was kann geschehen, um bei der Ausbildung (vor und nach Abschluß des Universitätsstudiums) das Verständnis der Juristen für psychologische, wirtschaftliche und soziologische Fragen in erhöhtem Maße zu fördern?" mit Gutachten von Eugen Ehrlich und Heinrich E. Gerlandt. Arthur Nußbaum propagierte und praktizierte „Rechtstatsachenforschung“. ${ }^{2}$ Von Ehrlich erschien 1913 eine „Grundlegung der Soziologie des Rechts“. Max Weber verfasste in den Jahren 1910-13 den postum in „Wirtschaft und Gesellschaft“ erschienenen Teil „Rechtssoziologie“. Damit ist nur ein Ausschnitt aus einer damals sehr umfangreichen Diskussion skizziert. Im ersten Weltkrieg scheint das interdisziplinäre Interesse geschwunden zu sein. Vielleicht lag es auch an der vehementen Kritik, die Hans Kelsen (zwischen 1915 und 1917) an Ehrlichs „Grundlegung“ geübt hatte, die dazu führte, dass sich Juristen wieder in ihrer rein normwissenschaftlichen Stube heimisch fühlen konnten. ${ }^{3}$

Es hat den Anschein, dass in der Weimarer Zeit Rechtssoziologie nur noch von einer kleinen Gruppe von akademisch nicht oder nicht sehr hoch etablierten Personen betrieben wurde. Diese wurden von Nazis aus Deutschland vertrieben. Rechtssoziologie sei zur Exil-Wissenschaft geworden. Erst in den 60er Jahren erwuchs neuerlich ein Interesse an der Rechtssoziologie (von juristischer und auch soziologischer Seite). Diese neuerliche Konjunktur brachte insbesondere bei den kritischen Juristen um die „Kritische Justiz“ eine Rückbesinnung auf die in der Weimarer Zeit begründete kritische Tradition der Rechtssoziologie. Um die Kater namentlich aus dem Sack zu lassen: Es handelte sich dabei um Hugo Sinzheimer und seine Schüler Ernst Fraenkel,

1 Mit dem berühmten Satz: „Dogmatik ohne Soziologie ist leer, Soziologie ohne Dogmatik blind.“ - Der Aufsatz ist enthalten in: Thomas Würtenberger (Hg.), Hermann Kantorowicz, Rechtswissenschaft und Soziologie. Ausgewählte Schriften zur Wissenschaftslehre (= Freiburger rechts- und staatswissenschaftliche Abhandlungen 19), Karlsruhe 1962, 117-144, 139.

2 Arthur Nußbaum, Deutsches Hypothekenwesen. Ein Lehrbuch, Tübingen 1913; ders., Die Rechtstatsachenforschung. Ihre Bedeutung für Wissenschaft und Unterricht (= Recht und Staat in Geschichte und Gegenwart 6), Tübingen 1914 (ND Berlin 1968).

3 Neuauflage der Texte mit einer Einführung von Klaus Lüderssen: Hans Kelsen, Eugen Ehrlich, Rechtssoziologie und Rechtswissenschaft. Eine Kontroverse (1915/17) (= Juristische Zeitgeschichte, Kleine Reihe 7), Baden-Baden 2003. - Vgl. zu dieser Diskussion bereits Hubert Rottleuthner, Rechtstheoretische Probleme der Soziologie des Rechts. Die Kontroverse zwischen Hans Kelsen und Eugen Ehrlich (1915/17), in: Rechtstheorie, Beiheft 5 (1984), 521-551, und auch Stanley L. Paulson (Hg.), Hans Kelsen und die Rechtssoziologie. Auseinandersetzungen mit Hermann U. Kantorowicz, Eugen Ehrlich und Max Weber, Aalen 1992. 
Franz Leopold Neumann und Otto Kahn-Freund. ${ }^{4}$ Hinzu kommt noch Otto Kirchheimer, der bei Carl Schmitt promoviert hatte. ${ }^{5} \mathrm{Zu}$ nennen sind weiter: Hermann Kantorowicz mit kleineren Beiträgen aus der Weimarer Zeit; ${ }^{6}$ Ludwig Bendix, der rechtssoziologisch relevant ist durch seine zahlreichen Beiträge zur richterlichen Urteilstätigkeit; ${ }^{7}$ Arthur Nußbaum führte seine Rechtstatsachenforschung fort; schließlich Theodor Geiger, der erst nach dem Zweiten Weltkrieg durch seine „Vorstudien zu einer Soziologie des Rechts“ in die Klassikerreihe der Disziplin einrückte, der aber bereits in den 20er Jahren einige rechtssoziologisch relevante Publikationen verfasste. ${ }^{8}$

Bei der ersten, der „Sinzheimer-Gruppe“ (also bei Sinzheimer, Fraenkel, Neumann und Kahn-Freund), handelte es sich um Rechtspraktiker (Rechtsanwälte, Richter Sinzheimer hatte als Rechtsanwalt eine Honorarprofessur inne). Ihre Tätigkeit auf arbeitsrechtlichem Gebiet versuchten sie theoretisch, d.h. sozialwissenschaftlich zu fundieren. ${ }^{9}$ Sie arbeiteten, und in dieser Hinsicht kommt dann Otto Kirchheimer hinzu, durchweg gewerkschaftsnah. Ludwig Bendix, Rechtsanwalt und Notar in Berlin, kam diesem Kreis als Mitbegründer und häufiger Autor der „Justiz“ nahe - seit 1925 das Organ des Republikanischen Richterbundes. Sinzheimer war von 1925 bis 1931 Mit-

4 Fraenkel und Kahn-Freund hatten bei Sinzheimer promoviert; Neumann bei Max Ernst Meyer (über „Staat und Strafe").

5 Otto Kirchheimer, Zur Staatstheorie des Sozialismus und Bolschewismus, Bonn 1928. Zum Verhältnis zwischen Otto Kirchheimer und Carl Schmitt vgl. Volker Neumann, Verfassungstheorien politischer Antipoden: Otto Kirchheimer und Carl Schmitt, KJ 14 (1981), 235-254.

6 Der Aufbau der Soziologie (1923), wieder abgedruckt in: Würtenberger (wie Anm. 1), 145-167, und die kurze Notiz: Max Weber (1922/23), in: ebd., 169-172.

7 Manfred Weiß (Hg.), Ludwig Bendix. Zur Psychologie der Urteilstätigkeit des Berufsrichters und andere Schriften (= Soziologische Schriften 43), Neuwied u.a. 1968.

8 Das uneheliche Kind und seine Mutter im Recht des neuen Staates. Ein Versuch auf der Basis kritischer Rechtsvergleichung, München u.a. 1920 (mit statistischem Material); Gesellschaft und Recht als Aufgaben der Volkshochschul-Lehre, Die Arbeitsgemeinschaft 4 (1923), 33-54; Soziologie der Industriearbeit und des Betriebs (1929), Neudruck in: Soziologische Texte 1 (1959), 147-167; Wirtschaft, Staat und Recht an der Volkshochschule, in: Freie Volksbildung, Neue Folge des Archivs für Erwachsenenbildung 1929, 43-46; Entwurf der Umrisse und Fragestellungen einer Soziologie des Rechts (1928), Wiederabdruck in: Theodor Geiger, Arbeiten zur Soziologie. Methode, moderne Großgesellschaft, Rechtssoziologie, Ideologiekritik, ausgewählt und eingeleitet von Paul Trappe (= Soziologische Texte 7), Neuwied u.a. 1962, 357-363.

9 Von Hugo Sinzheimer liegt aus der Weimarer Zeit an rechtssoziologisch Relevantem vor: Über soziologische und dogmatische Methoden in der Arbeitsrechtswissenschaft (1922), Wiederabdruck in: Otto Kahn-Freund/Thilo Ramm (Hg.), Hugo Sinzheimer. Arbeitsrecht und Rechtssoziologie. Gesammelte Aufsätze und Reden, Bd. 2 (= Schriftenreihe der Otto-BrennerStiftung 4), Frankfurt a.M. u.a. 1976, 33-41. - Von Ernst Fraenkel: Zur Soziologie der Klassenjustiz, Berlin 1927. Ansonsten sind die kritischen arbeitsrechtlichen Schriften hervorzuheben. Von Ernst Fraenkel: Kollektive Demokratie (1929), Zehn Jahre Betriebsrätegesetz (1930), Die politische Bedeutung des Arbeitsrecht (1932); von Franz L. Neumann: Die politische und soziale Bedeutung der arbeitsgerichtlichen Rechtsprechung (1929); von Otto KahnFreund: Das soziale Ideal des Reichsarbeitsgerichts (1931), Der Funktionswandel des Arbeitsrechts (1932). - All dies ist zu finden bei Thilo Ramm (Hg.), Arbeitsrecht und Politik. Quellentexte 1918-1933, Neuwied u.a. 1966. 
herausgeber der „Justiz““ ${ }^{10}$ Ab 1931 verfassten Sinzheimer und Fraenkel gemeinsam die „Chronik“ in der „Justiz“. Die anderen Personen (Kantorowicz, Nußbaum, Geiger) hatten akademische Positionen inne. Kantorowicz wurde nach seiner Habilitation 1908 zunächst, nämlich 1913, außerplanmäßiger Professor für ,juristische Hilfswissenschaften“. Erst 1929, mit 52 Jahren, erhielt er als Nachfolger von Radbruch und auf dessen massive Intervention hin eine ordentliche Professur in Kiel. ${ }^{11}$ Nußbaum kam bis 1933 nicht über den Status eines außerordentlichen Professors in Berlin hinaus. ${ }^{12}$ Theodor Geiger, noch 1918 als Jurist promoviert, brachte es 1929 zu einer ordentlichen Professur für Soziologie an der TH Braunschweig. ${ }^{13}$

Dies ist also die Gruppe, die ich näher analysieren möchte. Sie alle wurden aus Deutschland vertrieben, die meisten bereits 1933; Ernst Fraenkel verließ Deutschland (als letzter) erst 1938. Ich nehme schließlich noch Ernst Eduard Hirsch in meine Betrachtung auf. Er war zwar vor 1933 nicht rechtssoziologisch tätig, entwickelte sich aber in seinem türkischen Exil zum Rechtssoziologen und brachte nach seiner Rückkehr die Rechtssoziologie in der Bundesrepublik in den 60er Jahren entscheidend voran.

In meiner Liste fehlen zwei Autoren: Hans Kelsen, der sich zwar in seiner Kritik an Ehrlich vehement gegen dessen Versuch einer Begründung der Rechtssoziologie aus-

10 Von 1925 bis 1931 wurde sie herausgegeben von Wilhelm Kroner „in Verbindung mit“ Wolfgang Mittermaier, Gustav Radbruch und Hugo Sinzheimer. Ab 1932 tritt dann Karl Renner an die Stelle von Sinzheimer. Der erste Artikel im ersten Heft der „Justiz“ (1925, S. 6-11) stammt übrigens von Sinzheimer: „Zum Entwurf eines Arbeitsgerichtsgesetzes“.

$11 \mathrm{Zu}$ Kantorowicz vgl. Karlheinz Muscheler, Hermann Ulrich Kantorowicz. Eine Biographie (= Freiburger rechtsgeschichtliche Abhandlungen, NF 6), Berlin 1984; ders., Relativismus und Freirecht. Ein Versuch über Hermann Kantorowicz (= Freiburger rechts- und staatswissenschaftliche Abhandlungen 44), Heidelberg 1984; Monika Frommel, Hermann Ulrich Kantorowicz. Ein streitbarer Relativist, in: Kritische Justiz (Hg.), Streitbare Juristen. Eine andere Tradition, Baden-Baden 1988, 243-253; Sebastian Silberg, Hermann Kantorowicz und die Freirechtsbewegung, Berlin 2004; Frank Kantorowicz Carter [ein Sohn von Hermann Kantorowicz], Gustav Radbruch und Hermann Kantorowicz, in: German Law Journal vol. 7 no. 7 (1 July 2006). Diesem Beitrag ist übrigens zu entnehmen, dass der angeblich zweite Vorname „Ulrich“ später - von wem auch immer - angedichtet wurde.

12 Und wollte das als Anwalt wohl auch gar nicht. - Manfred Rehbinder behauptete in seinem Nachruf auf Nußbaum (JZ 20, 1965, 225 f.), dass er 1918 außerordentlicher Professor geworden sei, „später“ Ordinarius. Diese Behauptung hat er widerrufen in seinem Vorwort zur Ausgabe von Arthur Nußbaum, Die Rechtstatsachenforschung (= Schriftenreihe zur Rechtssoziologie und Rechtstatsachenforschung 12), Berlin 1968, 13, vielleicht aufgrund des Nachrufes von F. A. Mann, NJW 18 (1965), 577. Dort heißt es, dass Nußbaum 1920 außerordentlicher Professor geworden sei, nie aber ordentlicher Professor. Bei Ernst C. Stiefel/Frank Mecklenburg, Deutsche Juristen im amerikanischen Exil (1933-1950), Tübingen 1991, wird die Ernennung zum außerordentlichen Professor auf 1921 datiert. Auch ebd., 63, heißt es, dass er nie ordentlicher Professor geworden sei. - Zu Nußbaum vgl. auch den Gratulationsartikel zu seinem 80. Geburtstag, Columbia Law Review 57 (1957), 1-7.

13 Ein kleiner Exkurs: An der TH Braunschweig sollte Hitler 1932 eine außerordentliche Professur für „Organische Gesellschaftslehre und Politik“ erhalten, um dann - als deutscher Staatsbürger - bei der Reichspräsidentenwahl gegen Hindenburg antreten zu können. Der Senat der TH leistete erfolgreich Widerstand. Hitler wurde daraufhin von der braunschweigischen Regierung zum Regierungsrat ernannt (als „Sachbearbeiter für wirtschaftliche Fragen des Landes Braunschweig bei der Braunschweigischen Gesandtschaft in Berlin“), vgl. dazu Trappe (wie Anm. 8), 22. 
gesprochen hatte, ${ }^{14}$ was ihn aber nicht hinderte, die Soziologie als eine Seinswissenschaft, methodisch getrennt von der Rechtswissenschaft als einer Norm- oder Sollenswissenschaft, zu betreiben. Jedenfalls firmiert er noch in Kürschners Gelehrtenkalender von 1935 - da war er längst von seinem Kölner Lehrstuhl ins schweizerische Exil vertrieben - auch als Soziologe. Für die Rechtssoziologie wird man Kelsen vor allem mit seinen ideologiekritischen und wissenssoziologischen Beiträgen bewahren können. ${ }^{15}$ Der andere ist Karl Korsch ${ }^{16}$ - auch er Jurist, auf arbeitsrechtlichem Gebiet tätig, auch er wurde 1933 vertrieben. Aus nicht ganz klaren Gründen taucht er kaum in einer rechtssoziologischen Ahnenreihe auf. Vielleicht wurde er von den „Marxologen" der 60er und frühen 70er Jahre zu stark in Anspruch genommen für seine Fortentwicklung der Marxschen Theorie.

Bei all diesen Personen kann man allenfalls aus heutiger Sicht von „Rechtssoziologen" reden. Eine solche Stellen- oder gar Berufsbezeichnung gab es damals noch nicht. ${ }^{17}$ Aber sie haben rechtssoziologisch relevante Arbeiten veröffentlicht; manche, wie Sinzheimer, Kantorowicz oder Nußbaum, schon vor 1914. Auch bei den akademisch Tätigen orientiere ich mich bei der Identifizierung als „Rechtssoziologen“ nur an deren Veröffentlichungen, nicht an deren Stellenbeschreibungen. Über deren Lehrtätigkeit ist überdies nichts bekannt.

Wenn ich mich auf diese Personen konzentriere, heißt das nicht, dass es in der Zeit der Weimarer Republik nicht noch andere „Rechtssoziologen“ gegeben habe. In Betracht kommen Max Rumpf (1878-1953) mit einigen einschlägigen Veröffentlichun-

14 Vgl. oben Anm. 3.

15 Vgl. Paulson (wie Anm. 3) mit den eher rechtssoziologiekritischen Beiträgen Kelsens (zu Kantorowicz, Ehrlich und Max Weber).

16 1886-1961; 1912 Dr. iur. in Frankfurt a.M.; 1919 USPD, 1920-26 KPD; Professor für Arbeitsrecht in Jena; 1933 Emigration nach England, Holland, 1936 USA. - Was ist Sozialisierung? (1919); Arbeitsrecht für Betriebsräte (1922); Marxismus und Philosophie (1923); Um die Tariffähigkeit. Eine Untersuchung über die heutigen Entwicklungstendenzen der Gewerkschaftsbewegung 1928; Die materialistische Geschichtsauffassung (1929); Karl Marx, London 1938. Die meisten dieser Arbeiten erschienen nach 1966 als graue Reprints. Vgl. auch: Ius belli ac pacis im Arbeitsrecht, KJ 5 (1972), 142-149, mit Anmerkungen zu Korschs Rechtstheorie von Jürgen Seifert, 149-153. Seit 1978 wird die Korsch-Gesamtausgabe von Michael Buckmiller herausgegeben im Auftrag des Internationalen Instituts für Sozialgeschichte IISG in Amsterdam und des Instituts für Politische Wissenschaft der Universität Hannover. Die oben genannten Arbeiten sind, mit Ausnahme von „Um die Tariffähigkeit“ und „Karl Marx“, enthalten in: Michael Buckmiller (Hg.), Karl Korsch. Gesamtausgabe, Bd. 2: Rätebewegung und Klassenkampf. Schriften zur Praxis der Arbeiterbewegung 1919-1923, Hannover 1980; Bd. 3: Marxismus und Philosophie. Schriften zur Theorie der Arbeiterbewegung 1929-1933, Hannover 1993; Bd. 5: Krise des Marxismus, Hannover 1996.

17 Sinzheimer hatte an der Frankfurter Universität eine Honorarprofessur für Arbeitsrecht. 1929 erhielt er einen unbesoldeten Lehrauftrag für Soziologie unter besonderer Berücksichtigung der Rechtssoziologie. So ist er auch seit dem Sommersemester 1930 im Vorlesungsverzeichnis aufgeführt. Vgl. dazu Hans-Peter Benöhr, Hugo Sinzheimer (1875-1945), in: Bernhard Diestelkamp/Michael Stolleis (Hg.), Juristen an der Universität Frankfurt a.M., Baden-Baden 1989, 67-83, 70 ff. - In Kürschners Gelehrtenkalender von 1931 ist er nur mit dem Fach Arbeitsrecht verzeichnet. 
gen ${ }^{18}$ und Franz Wilhelm Jerusalem (1883-1970) mit seinem ersten Band einer „Soziologie des Rechts. Gesetzmäßigkeit und Kollektivität" von 1925, dem kein zweiter folgte. Als Jurist, der sich - wie Kelsen - im Kürschner zugleich als Soziologe verzeichnen ließ, wäre noch Carl August Emge (1886-1970) zu nennen. Der war bei aller bunten Vielfalt aber eher in rechtsphilosophischen Gefilden engagiert. Max Rumpf regredierte schon vor 1933 zu einer bodenständigen Volkskunde, vor allem des Bauernstandes - ohne Rechtsbezug. Jerusalem, der nach 1933 den Ariernachweis erbringen konnte, veröffentlichte nichts rechtssoziologisch Relevantes mehr. Man wird also sagen können, dass die Rechtssoziologie nach 1933 zu einer „Exilwissenschaft“ wurde. Das heißt aber nicht, dass in der NS-Zeit keine rechtssoziologisch relevanten Untersuchungen angestellt wurden, wie z.B. zur Wirkung von Gesetzen, zu den Einstellungen der Bevölkerung zu Recht und Justiz etc. ${ }^{19}$

Ich möchte nun für die in die Auswahl aufgenommenen Personen folgende Fragen beantworten:

Was waren die Merkmale dieser rechtssoziologisch relevanten Autoren vor 1933? Wann gingen sie wohin ins Exil? Was machten sie im Exil? Was machten sie nach 1945?

\section{Was waren die Merkmale dieser rechtssoziologisch relevanten Autoren vor 1933 ?}

Was waren die gemeinsamen Merkmale unserer Autoren? Überflüssig zu sagen: alle waren männlich. Allerdings: Über ihre Frauen und Kinder, wenn sie denn welche hatten, erfahren wir üblicherweise (fast) nichts. ${ }^{20}$ Alle hatten eine juristische Ausbildung absolviert, Geiger anscheinend nur bis zum Ersten Staatsexamen. Alle wurden promoviert. Mit Ausnahme von Geiger waren alle jüdischer Abstammung. Die meisten waren Mitglied der SPD. Von Bendix und Nußbaum ist mir keine Parteizugehörigkeit bekannt. Kantorowicz war nur ein Jahr (1903) in der SPD, in der Weimarer Zeit war er Mitglied der DDP. ${ }^{21}$ Geiger trat 1918 in die SPD ein, verließ sie aber aus Protest gegen deren Politik im September 1932. Viele waren für die Gewerkschaft und/oder in der Arbeiterbildung tätig, etwa an der Akademie der Arbeit in Frankfurt a.M. und

18 Was ist Rechtssoziologie?, AcP 122 (1924), 36-51; Anwalt und Anwaltstand. Eine rechtswissenschaftliche und rechtssoziologische Untersuchung (= Druckschriften Deutscher Anwaltverein 8), Leipzig 1926; Rechtswissenschaft als Sozialwissenschaft? Antrittsvorlesung (= Nürnberger Beiträge zu den Wirtschaftswissenschaften 17), Nürnberg 1929.

19 Vgl. dazu Hubert Rottleuthner, Rechtsphilosophie und Rechtssoziologie im Nationalsozialismus, in: Ralf Dreier/Wolfgang Sellert (Hg.), Recht und Justiz im „Dritten Reich“, Frankfurt a.M. 1989, 295-322.

20 Ausnahme: Die Autobiographie von Reinhard Bendix, dem Sohn von Ludwig Bendix: Von Berlin nach Berkeley. Deutsch-jüdische Identitäten, Frankfurt a.M. 1985. Der erste Teil behandelt die Lebensgeschichte seines Vaters bis 1935. - Autobiographien unserer Autoren sind selten: Otto Kahn-Freund, Autobiographische Erinnerungen an die Weimarer Republik. Ein Gespräch mit Wolfgang Luthardt, KJ 14 (1981), 183-200. - Ernst E. Hirsch, Aus des Kaisers Zeiten durch die Weimarer Republik in das Land Atatürks. Eine unzeitgemäße Biographie, München 1982, gekürzte Neuauflage: Als Rechtsgelehrter im Lande Atatürks, Berlin 2008.

21 Frank Kantorowicz Carter (wie Anm. 11), 13. 
der Hochschule für Politik in Berlin 22 (der Sinzheimer-Kreis, Kirchheimer, Geiger). Einige Autoren waren Mitglieder des Republikanischen Richterbundes und über dessen Organ „Die Justiz“ miteinander verbunden. Sie waren teils Rechtsanwälte (Fraenkel und Neumann betrieben ab 1928 gemeinsam eine Kanzlei in Berlin), teils waren sie an Universitäten tätig. Nur einer, Kahn-Freund, war Richter. Von den akademisch Tätigen hatten Kantorowicz und Geiger eine ordentliche Professur inne; Kantorowicz aber - wie gesagt - erst mit 52 Jahren; Geiger bei den Soziologen. Sinzheimer hatte eine Honorarprofessur, Nußbaum blieb bis 1933 außerordentlicher Professor. Vom Fach her standen damalige Randgebiete im Vordergrund: das Arbeitsrecht beim Sinzheimer-Kreis und auch bei Bendix; ${ }^{23}$ das auch noch junge Wirtschaftsrecht bei Nußbaum, Rechtsgeschichte bei Kantorowicz.

Ich möchte hier von einem Marginalitäts-Syndrom sprechen: denn hinsichtlich der politischen Orientierung, der Konfession, des Arbeitsgebietes, der akademischen Position sind die genannten Personen randständig.

Beim Merkmal „Jude“ lässt sich unterscheiden, ob es sich um ein ,Verfolgungsmerkmal' handelt, das von den Nazis zur administrativen Handhabbarkeit in vielen Verordnungen definiert wurde. Damit lässt sich die Exilierung der Betroffenen erklären. Als ,Erklärungsmerkmal' für Einstellungen und Verhaltensweisen ist es nur begrenzt tauglich. Gibt es eine enge Korrelation zwischen dem Judesein und der Art der Tätigkeit und/oder der politischen Orientierung? Erforderlich wäre in diesem Erklärungszusammenhang allererst eine Abstufung des Merkmals „Jude“, z.B. von überzeugten, praktizierenden Juden über „Drei-Tage-Juden“ (ähnlich den „Christen“, die Weihnachten, Silvester und Ostern in die Kirche gehen), durch christliche Taufe assimilierte Juden bis zu vehement antireligiösen „Juden“, denen dieses Etikett erst von den Nazis angeheftet wurde. ${ }^{24}$

Auf einen wesentlichen Unterschied in der inhaltlichen Orientierung dieser rechtssoziologischen Gruppierung möchte ich hinweisen. Ehrlich ging - wie dann auch Nußbaum und Kantorowicz - von der Diskrepanz zwischen staatlichem Gesetzesrecht und dem tatsächlichen, rechtsbezogenen sozialen Handeln aus, kurz: von dem Widerspruch zwischen „Recht und Leben“. Die Freirechtler Ehrlich und Kantorowicz betonten überdies die relativ eigenständige Rolle des Richters gegenüber dem Gesetz. Sinzheimer legte zwar auch größten Wert darauf, die tatsächlichen Verhältnisse im Arbeitsleben zu erforschen, insbesondere die sich herausbildendenden „korporativen Arbeitsnormenverträge". Er und seine Schüler waren aber keine Freirechtler. Sie begriffen das entstehende Recht der abhängigen Arbeit als eine grundsätzliche Herausforderung für das bürgerliche Formalrecht, das von der Autonomie gleicher Subjekte ausgeht. Sie wollten, ohne Marxisten zu sein, sozialpolitisch ein neues Recht, eben ein Recht der abhängigen Arbeit gestalten - und dies hatte vor allem Gesetzesrecht zu sein. Die

22 Die HfP wurde 1920 gegründet, 1933 in ihrer alten Form geschlossen und am 15. Januar 1949 wieder eröffnet, vgl. Ernst Jäckh/Otto Suhr, Geschichte der Deutschen Hochschule für Politik, Berlin 1952.

23 Ludwig Bendix war Fachanwalt für Arbeitsrecht und nebenamtlicher Vorsitzender beim Arbeitsgericht Berlin, vgl. Reinhard Bendix (wie Anm. 20), 195. - Für „Die Justiz“ verfasste er eine Fülle von Beiträgen bis 1933.

$24 \mathrm{Zu}$ der letzten Gruppe vgl. etwa die Autobiographie von Peter Gay, Meine deutsche Frage. Jugend in Berlin 1933-1939, München 1999. 
Herausbildung eines neuen Arbeitsrechts konnte man nicht einer Richterschaft überlassen, über deren Zusammensetzung und politische Orientierung sich Sinzheimer vor 1918 im klaren war; und diese Einschätzung zu revidieren hatte sein Kreis nach 1918 angesichts der Konstanz der Richterschaft keinen Anlass. ${ }^{25}$

\section{Wann gingen sie wohin ins Exil?}

Die meisten, nun einschließlich Ernst E. Hirsch, verließen Deutschland bereits 1933. Nußbaum ging 1934 in die USA, nachdem er im September 1933 zwangsweise in den Ruhestand versetzt worden war. Ludwig Bendix verließ erst 1937 Deutschland, nach einigen Monaten Inhaftierung 1933 und fast zwei Jahren „Schutzhaft“ zuletzt in Dachau. ${ }^{26} \mathrm{Ihm}$ war danach auferlegt worden, Deutschland innerhalb von 14 Tagen Richtung Palästina zu verlassen. Nur mit Mühe konnte er von seiner Familie abgehalten werden, in seinem noch immer ungebrochenen Legalitätsglauben Strafanzeige gegen den Kommandanten von Dachau zu erstatten. ${ }^{27}$ Als letzter verließ Fraenkel das gesunkene Schiff am 20. September 1938 - eine Woche bevor Juden der Beruf des Rechtsanwalts endgültig verschlossen wurde. ${ }^{28}$ Nach wenigen Wochen Aufenthalt in London bei Kahn-Freund fuhr er noch 1938 in die USA.

Die meisten unserer Autoren gingen in die USA, Neumann mit langer Zwischenstation in England, Kirchheimer in Frankreich. Kantorowicz ging 1935 von den USA

$25 \mathrm{Zu}$ Sinzheimer und auch zu seinen Schülern vgl. Sandro Blanke, Soziales Recht oder kollektive Privatautonomie? Hugo Sinzheimer im Kontext nach 1900 (= Beiträge zur Rechtsgeschichte des 20. Jahrhunderts 46), Tübingen 2005. - Im Brustton der Überzeugung, die wahren Interessen der Arbeiterklasse zu kennen, polemisierte Karl Korsch über die „opportunistisch-reformistische“, „unmarxistische“ Auffassung von Hugo Sinzheimer, die er in seinem Vortag „,Das zukünftige Arbeitsrecht in Deutschland“ auf dem Leipziger Gewerkschaftskongress 1922 vorgetragen hatte, vgl. Karl Korsch, Der tote Sinzheimer und der lebende Marx (1922), in: Gesamtausgabe Bd. 2 (wie Anm. 16), 537-543.

26 Vom 30.7.1935 bis zum 22.5.1937; vgl. Reinhard Bendix (wie Anm. 20), 259.

27 Reinhard Bendix (wie Anm. 20), $270 \mathrm{ff}$.

28 Durch die 5. VO zum Reichsbürgergesetz vom 27.9.1938, RGBl. I, 1403. Zum Datum der Flucht vgl. Alexander von Brünneck, Vorwort, in: Ders. (Hg.), Ernst Fraenkel. Gesammelte Schriften, Bd. 2, Baden-Baden 1999, 9. - In einem Dokument der Gestapo von 1939 taucht Fraenkel als Dr. phil. auf, ebd., 641. 
nach England (Cambridge). ${ }^{29}$ Sinzheimer erhielt 1933 in Amsterdam und Leiden eine Honorarprofessur für Arbeitsrecht und Rechtssoziologie. Geiger fasste in Dänemark Fuß aufgrund seiner guten Kenntnisse der skandinavischen Sprachen. Hirsch, der sich 1930 für Handelsrecht, Bürgerliches Recht, Deutsches und Internationales Privatrecht habilitiert hatte, ${ }^{30}$ suchte in der Türkei Zuflucht. Ihm war an der reformierten Universität Istanbul eine Professur für Handels- und Seerecht angeboten worden. In kurzer Zeit lernte er türkisch. ${ }^{31}$ Noch heute spricht man dort groß von Ernst Hirsch. Die Sprachbarriere, die das Exil mit sich brachte, ließ sich also irgendwie überwinden. Viel schwieriger aber war es, zwei andere Probleme des Exils zu bewältigen: ${ }^{32}$ das Vertrautwerden mit einer anderen Rechtskultur; für Juristen ist das besonders schwierig, soweit sie in einer nationalen Rechtsordnung ausgebildet wurden. Eigentlich müssten sie noch einmal ein neues Studium beginnen. Die meisten der Exilierten waren zudem mit einem weiteren Problem konfrontiert: die Verarbeitung ihrer Niederlage als homo politicus.

Auf die Rechtsordnung des Gastlandes haben sich nur wenige eingelassen. Als Soziologe war Geiger solcher Probleme enthoben. Keine großen Schwierigkeiten hatte wohl Kantorowicz als Rechtshistoriker und Nußbaum als Kenner des IPR und der Rechtsvergleichung. Hirsch kam die Rezeption des westeuropäischen Rechts in der Türkei Atatürks zugute. Zugleich konnte er sich als Experte des westlichen Handelsrechts bei dessen Adaption profilieren. Ansonsten hat sich anscheinend nur Kahn-

29 Nach Kürschners Gelehrtenkalender 1935 (in dem er noch erwähnt wird!) hatte Kantorowicz eine Gastprofessur in Florenz, ging 1933/34 an die New School, war 1934 Lecturer am College of the City of New York, 1934/35 an der LSE, 1935 University Cambridge. Nach Muscheler (wie Anm. 11), $106 \mathrm{ff}$. lässt sich ergänzen, dass Kantorowicz zum 26.9.1933 endgültig aus dem Hochschuldienst entlassen wurde. Zuvor war er schon nach Cambridge gegangen (er hatte aufgrund von Vortragreisen in den Jahren 1924, 1926 und 1931 gute Kontakte in England), dann aber gleich an die „University in Exile“ (,The Graduate Faculty of Political and Social Science") an der 1919 gegründeten New School for Social Research, wo er bis Mitte 1934 blieb. Im April 1934 hielt er an der Columbia University bei Llewellyn den bekannten Vortrag „Some Rationalism about Realism“. Ab Juni 1934 war er zunächst an der London School of Economics tätig, ab 1935 vor allem in Cambridge, aber auch in Oxford und Glasgow. - Von Interesse sind auch die Bemerkungen von Muscheler (wie Anm. 11), 111 ff., zur Gründung des Institut International de Philosophie du Droit et de Sociologie Juridique am 7.-10. Oktober 1933 in Paris. Dieses Institut war durchaus als Konkurrenz zur von Deutschen dominierten Internationalen Vereinigung für Rechts- und Wirtschaftsphilosophie (ab 1933: für Rechts- und Sozialphilosophie, IVR) gedacht. Präsident war Louis le Fur, Vizepräsidenten u.a. Kelsen und Sinzheimer. Kantorowicz und sein Freund Radbruch erhielten den Status als Membres Titulaires.

30 Seine arbeitsrechtlichen Habilitationsleistungen wurden übrigens von Sinzheimer kritisiert, so dass er dafür keine venia erhielt, vgl. Benöhr (wie Anm. 17), $75 \mathrm{f}$.

31 Vgl. Ernst E. Hirsch. Liber Amicorum. Eine Bio- und Bibliographie anlässlich seines 75. Geburtstages, Amriswil 1977, 44-49. - Von 1943 bis 1952 hatte er eine Professur für Handels-, See- und Versicherungsrecht, Urheber- und Patentrecht, Rechtsphilosophie und Rechtssoziologie in Ankara inne. Ein türkisches Lehrbuch der Rechtsphilosophie und Rechtssoziologie wurde 1949 veröffentlicht: Ernest Hirs, Hukuk Felsefesi ve Hukuk Sosyolojisi Dersleri, Ankara 1949.

32 Vgl. Franz Neumann, Intellektuelle Emigration und Sozialwissenschaft, in: Alfons Söllner (Hg.), Franz Neumann. Wirtschaft, Staat, Demokratie. Aufsätze 1930-1954, Frankfurt a.M. $1978,402-423$. 
Freund ab ovo auf das Recht des Gastlandes eingelassen. Er machte 1935 seinen Master of Laws (LL.M.) an der London School of Economics and Political Science (LSE).

\section{Was machten sie im Exil?}

Wie überlebt man in der Fremde? Stipendien zur Weiterqualifikation waren rar. Ein Auskommen fand man als Honorarprofessor, Gastprofessor oder Forschungsprofessor an Universitäten - allen voran an der Columbia University mit ihrem Zweig der „New School“. Daneben gab es Forschungsstellen am exilierten Institut für Sozialforschung, etwa für Neumann und Kirchheimer. Eine „richtige“ Professur erhielten Geiger und Hirsch. Im Krieg, nach 1942, engagierten sich Fraenkel, Neumann und Kirchheimer (übrigens auch Herbert Marcuse) in US-Regierungsstäben als Deutschland-Experten für die kommende Okkupation. ${ }^{33}$

Im Exil blieb nur Kahn-Freund seinem Metier, dem Arbeitsrecht, treu. ${ }^{34}$ Ein Jahr nachdem er an der LSE seinen LL.M. gemacht hatte (1935), erwarb Franz Neumann ebenfalls an der LSE einen Ph.D. für eine politikwissenschaftliche Arbeit bei Harold Laski $^{35}$ (,The Governance of the Rule of Law"). ${ }^{36}$ Fraenkel absolvierte in Chicago ein Jura-Studium (u.a. bei Professor Ehrlicher, einem Onkel von Kahn-Freund), war kurz als Anwalt tätig, konnte aber anscheinend in seinem alten Beruf in der neuen Welt nicht reüssieren. ${ }^{37} \mathrm{Ihr}$ Lehrer Sinzheimer widmete sich im holländischen Exil nun vorrangig der Rechtssoziologie und befasste sich auch mit Marx. ${ }^{38}$ Was sollte er auch noch über Arbeitsrecht schreiben? Es war Kahn-Freund, der die arbeitsrechtliche Tradition fortsetzte. Was Sinzheimer für Deutschland vollbrachte, die arbeitsrechtliche Durchdringung einer Tarifvertragsordnung (,korporative Arbeitsnormenverträ-

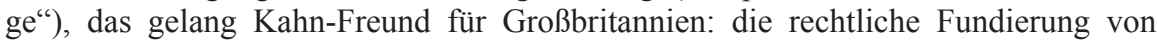
„,collective agreements“. 39

33 Vgl. Alfons Söllner (Hg.), Zur Archäologie der Demokratie in Deutschland, Bd. 1: Analysen politischer Emigranten im amerikanischen Geheimdienst 1943-1945, Frankfurt a.M. 1982.

34 Er war bereits 1927 und 1928 in England und Amerika und kam, wie er sagte, mütterlicherseits aus einer „internationalen Familie“, vgl. Kahn-Freund (wie Anm. 20), 187. „Ich war von Beginn an der Ansicht, wenn ich Deutschland verlasse, verlasse ich Deutschland nicht als Emigrant, sondern als Immigrant. ... Ich wollte englischer Jurist werden“, ebd., 191.

35 Dem Kahn-Freund schon 1928 begegnet und mit dem er später befreundet war, vgl. KahnFreund (wie Anm. 20), 184, 191.

36 Deutsche Übersetzung von Alfons Söllner: Franz Neumann, Die Herrschaft des Gesetzes. Eine Untersuchung zum Verhältnis von politischer Theorie und Rechtssystem in der Konkurrenzgesellschaft, Frankfurt a.M. 1980.

37 Vgl. dazu Simone Ladwig-Winters, Ernst Fraenkel als Stipendiat des American Committee in Chicago, in: Hubertus Buchstein/Gerhard Göhler (Hg.), Vom Sozialismus zum Pluralismus. Beiträge zu Werk und Leben Ernst Fraenkels, Baden-Baden 2000, 43-61.

38 Die Aufgabe der Rechtssoziologie (1935); Der Hintergrund des Arbeitsrechts (1936); Eine Theorie des sozialen Rechts (1936); Der junge Marx und die Soziologie des Rechts (1937); Das Transformationsproblem in der Soziologie des Rechts (1938); Theorie der Gesetzgebung. - Alles in: Sinzheimer (wie Anm. 9).

39 Vgl. auch seine entsprechenden Bemerkungen, KJ 14 (1981), 187. 
Die kurze Berührung mit dem amerikanischen „realism“ (Holmes, Pound u.a.) führte bei Kantorowicz nur zu einem kleinen, aber gewichtigen rechtssoziologisch einschlägigen Aufsatz (,Some Rationalism About Realism“, 1934). ${ }^{40}$ Dagegen bildete die ausführliche Beschäftigung Geigers mit dem skandinavischen Rechtsrealismus (Hägerström, Lundstedt, Olivecrona, Ross u.a.) das Fundament, auf dem er seine ,Vorstudien zu einer Soziologie des Rechts“ schreiben konnte (veröffentlicht 1947) - keine empirische Rechtssoziologie, sondern eine rechtstheoretische Grundlegung einer Rechtssoziologie. ${ }^{41}$ Daneben widmete er sich im dänischen, dann (1943) schwedischen Exil einer Fülle weiterer allgemein soziologischer Themen. ${ }^{42}$

Hirsch wurde in der Türkei überhaupt erst zum Rechtssoziologen aufgrund einer eben rechtssoziologischen Grunderfahrung: der Diskrepanz von geschriebenem, in der Türkei rezipiertem fremden Recht und dem in einer Gesellschaft „lebenden Recht““.

Der hervorstechende Befund aber ist der: die sozialwissenschaftlich erhellten Aktivisten eines ,linken“ Arbeitsrechts der Weimarer Zeit - Fraenkel, Neumann und Kirchheimer - wandelten sich im Exil zu Politikwissenschaftlern. ${ }^{43}$ Sie befassten sich zwar noch mit dem Untergang von Weimar. Bedeutsam für die rechtssoziologische und politikwissenschaftliche Tradition, die hier nicht zu trennen sind, wurden aber ihre Analysen des nationalsozialistischen Herrschaftssystems. Fraenkel stellte in der Phase der „inneren Emigration“, ${ }^{44}$ d.h. bis er 1938 Deutschland verlassen musste, die Urfassung des „Doppelstaates“ fertig. Die englische Fassung erschien dann 1940/41 im amerika-

40 Ursprünglich in Yale Law Journal 43 (1934), 1240 ff.; deutsche Übersetzung: Rationalistische Bemerkungen über Realismus, in: Kantorowicz (wie Anm. 1), 101-116.

41 Mit dem Nationalsozialismus hat er sich darin wohl nur an einer Stelle befasst: „Die Herrschaftsträger können natürlich den einzelnen Richter vergewaltigen, weil er ökonomisch und mit seiner ganzen Existenz von ihnen abhängig ist. Aber sie können nicht das Richterkorps als ganzes zur Gefügigkeit zwingen. Wenn dieses sich in seiner Sanktionstätigkeit an die bestehenden Gesetze hält und auf sie pocht, in denen ,die Staatsmacht' sich selbst gebunden hat, dann ist die Staatsmacht gebunden. Darin liegt die unauslöschliche Schuld des deutschen Richterstandes unter Hitler: dass er allzuwenige Persönlichkeiten zählte, an deren um persönliche Gefahr unbekümmerter Standhaftigkeit kollektiver Widerstand des Richterstandes als ganzen sich hätte emporranken können. Stattdessen erwies sich der Stand als ein Heer depravierter Kreaturen der brutalen Gewalt." Vgl. Manfred Rehbinder (Hg.), Theodor Geiger, Vorstudien zu einer Soziologie des Rechts (= Schriftenreihe zur Rechtssoziologie und Rechtstatsachenforschung 65), 4. Aufl. Berlin 1987, 338.

42 Aus dieser Zeit stammt auch seine Kritik an der rechtstheoretischen Uppsala-Schule des skandinavischen Rechtsrealismus; vgl. dazu seine Beiträge in: Über Moral und Recht. Streitgespräch mit Uppsala (= Schriftenreihe zur Rechtssoziologie und Rechtstatsachenforschung 45), Berlin 1979. Mit Recht wurde kritisiert, dass in Jes Bjarups Übersicht Skandinavischer Realismus: Hägerström, Lundstedt, Olivecrona, Ross, Freiburg 1978, Geiger überhaupt nicht auftaucht.

43 Fraenkel behauptet in seiner Gedenkrede für Neumann, dass dieser eigentlich schon durch seine Weimarer Aktivitäten zu einem Politologen gemacht worden sei, vgl. Ernst Fraenkel, Gedenkrede auf Franz L. Neumann (1955), in: Falk Esche/Frank Grube (Hg.), Ernst Fraenkel, Reformismus und Pluralismus, Hamburg 1973, 168-179, 176.

44 Befand er sich bis 1938 in der ,inneren Emigration“? Für die behauptete Neumann in seinem Aufsatz von 1952, dass die Schreibtische der inneren Emigranten leer geblieben seien, vgl. Intellektuelle Emigration und Sozialwissenschaft, in: Söllner (wie Anm. 32), 402-423, 409. Fraenkels Berliner Schreibtisch war gewiss nicht leer geblieben; er schrieb überdies für die Exil-Zeitschrift „Sozialistische Warte“, Paris 1934-39. 
nischen Exil. Ein Jahr später (1942) wurde die 1. Auflage von Neumanns „Behemoth“ publiziert, 1944 die 2. Auflage. Diese beiden Bücher beziehen sich auf unterschiedliche Phasen des NS-Systems, was sich in den Grundthesen von einem „Doppelstaat“ bei Fraenkel und von einem „non-state“ bei Neumann niederschlägt. Methodisch ist das Erstaunliche an diesen beiden grundlegenden, theoretisch ambitionierten und empirisch gesättigten Arbeiten, dass hier ohne Zugriff auf irgendein Archivmaterial, also in den Grenzen einer reinen „Fassadenforschung“, tiefgründige Einsichten über die Herrschaftspraxis des Nationalsozialismus vermittelt werden können. - Das gilt auch für drei Aufsätze von Kirchheimer über Staatsgefüge und Recht im „Dritten Reich“, das Strafrecht im nationalsozialistischen Deutschland und die Rechtsordnung des Nationalsozialismus. ${ }^{45}$

Angesichts dieses Wandels zu Politikwissenschaftlern sind unterschiedliche Formen der Traditionspflege aufschlussreich. Im Juristenlexikon von Stolleis ${ }^{46}$ werden als „Juristen“ in die Jahrhunderte umspannende Galerie aufgenommen: Sinzheimer, Kahn-Freund und Kantorowicz. Die anderen kommen nicht vor. Im Band der „Kritischen Justiz“ über „Streitbare Juristen“47 tauchen Sinzheimer und sein gesamter Kreis wie auch Kirchheimer auf. Es fehlt aber der so streitbare Bendix, es fehlen die anscheinend nicht so streitbaren Nußbaum, Geiger und Hirsch.

\section{Was machten sie nach 1945?}

Im Exil verstarben Kantorowicz (1940) und Sinzheimer (September 1945). Zurückgekehrt sind nur Fraenkel und Hirsch, beide nach Berlin. Der eine, Fraenkel, zu den Politikwissenschaftlern an der Hochschule für Politik, dann das Otto-Suhr-Institut. 1953 erhielt er dort eine Professur, erst 1961 eine ordentliche. Später engagierte er sich bei der Gründung des John-F.-Kennedy-Instituts für Amerikanistik an der Freien Universität (FU), 1964. Der andere, Ernst-E.-Hirsch, wurde Mitglied des Fachbereichs Rechtswissenschaft der FU. Hirsch hatte bereits seit 1948 regelmäßig eine Gastprofessur in Westdeutschland und Berlin wahrgenommen, u.a. auch mit rechtssoziologischen Vor-

45 Staatsgefüge und Recht des dritten Reiches (pseudonym Dr. Hermann Seitz), angeblich in Hamburg 1935 bei der Hanseatischen Verlagsanstalt als Heft 12 der Schriftenreihe „Der deutsche Staat der Gegenwart" erschienen, tatsächlich wurde es in Paris 1935 hergestellt, vgl. dazu auch die empörte Reaktion - vielleicht von Carl Schmitt - in DJZ 40 (1935), Sp. 1104 f., wieder abgedruckt in: Wolfgang Luthardt (Hg.), Otto Kirchheimer, Von der Weimarer Republik zum Faschismus, Frankfurt a.M. 1976, 152-185, und bereits in: KJ 9 (1976), 33-59, mit einer Einleitung von Wolfgang Luthardt. - Das Strafrecht im nationalsozialistischen Deutschland (Original: Criminal Law in National-Socialist Germany 1939/40), in: Kirchheimer, ebd., 186-212. - Die Rechtsordnung des Nationalsozialismus (Original: The Legal Order of National Socialism, 1941), in: Funktionen des Staates und der Verfassung, Frankfurt a.M. 1972, 115-142. - Daneben arbeitete Kirchheimer gemeinsam mit Georg Rusche an: Punishment and Social Structure, New York 1939 (dt. Sozialstruktur und Strafvollzug, Frankfurt a.M. 1974).

46 Michael Stolleis (Hg.), Juristen. Ein biographisches Lexikon. Von der Antike bis zum 20. Jahrhundert, München 1995.

47 Streitbare Juristen (wie Anm. 11). 
lesungen. ${ }^{48} 1952$ wurde er ordentlicher Professor an der FU für Handelsrecht, Bürgerliches Recht und Rechtsphilosophie; er hielt regelmäßig Vorlesungen zur Rechtssoziologie. ${ }^{49} 1964$ gründete er das Institut für Rechtssoziologie und Rechtstatsachenforschung. Von 1966 bis 1968 erschien die „Schriftenreihe des Instituts für Rechtssoziologie und Rechtstatsachenforschung“, herausgegeben von Ernst-E.-Hirsch (Band 1: „Das Recht im sozialen Ordnungsgefüge“ mit seinen gesammelten Beiträgen). Im Geleitwort zu der Reihe platzierte er sich in der Tradition von Ehrlich und Nußbaum. ${ }^{50}$ 1967 erschien - herausgegeben von Hirsch und seinem Assistenten Manfred Rehbinder, ohne den weder Institut noch Schriftenreihe zur Existenz gekommen wären - das Sonderheft 11 „Rechtssoziologie“ der Kölner Zeitschrift für Soziologie und Sozialpsychologie. Damit erhielt die Rechtssoziologie in der Bundesrepublik einen entscheidenden Anschub. Seit 1968, ab Band 12, nach der Emeritierung von Hirsch, erscheint die Schriftenreihe unter dem geänderten Titel „Schriftenreihe zur Rechtssoziologie und Rechtstatsachenforschung", herausgegeben von Ernst-E.-Hirsch bis zu seinem Tod 1985 und ab 1970, mit Band 18, auch und ab 1985 nur von Manfred Rehbinder, der sich 1968 an der FU Berlin in den Fächern Rechtssoziologie, Handelsrecht und Arbeitsrecht habilitiert hatte.

Bendix, Geiger, Kahn-Freund, Kirchheimer, Neumann und Nußbaum blieben im Exil - durchweg auf akademischen Positionen, mit Ausnahme von Ludwig Bendix, der 1947 im Alter von 70 Jahren von Palästina zu seiner Familie in die USA zog. KahnFreund machte die Karriere, die er machen wollte: als englischer Jurist. ${ }^{51}$ Er wurde zum führenden Vertreter des englischen Arbeitsrechts. An seine Weimarer Zeit hat er sich in mehreren Beiträgen literarisch erinnert. ${ }^{52}$ Er veröffentlichte weiter rechtssozio-

48 Vgl. Liber Amicorum (wie Anm. 31), 16 f.

49 Vgl. das Skript zur Vorlesung in: Ernst E. Hirsch, Das Recht im sozialen Ordnungsgefüge. Beiträge zur Rechtssoziologie ( = Schriftenreihe des Instituts für Rechtssoziologie und Rechtstatsachenforschung 1), Berlin 1966, $315 \mathrm{ff}$.

50 Hirsch (wie Anm. 49), 5. - Vgl. auch seine frühen Beiträge: Rechtssoziologische Stichworte, in: Wilhelm Bernsdorf/Friedrich Bülow (Hg.), Wörterbuch der Soziologie, Stuttgart 1955; Die Rezeption fremden Rechts als sozialer Prozeß, in: Otto Stammer/Karl C. Thalheim (Hg.), Festgabe für Friedrich Bülow zum 70. Geburtstag, Berlin 1960, 121-137; Was kümmert uns die Rechtssoziologie?, Juristenjahrbuch 3 (1962/63), 131-148. Die beiden letztgenannten Beiträge sind in dem Sammelband von 1966 enthalten. - Eugen Ehrlichs Grundlegung der Soziologie des Rechts erschien in der 3. Aufl. Berlin 1967; 1968 gab Manfred Rehbinder Arthur Nußbaum, „Die Rechtstatsachenforschung“ in der Schriftenreihe des Instituts heraus und versah sie mit einer Einleitung.

51 Vgl. Anm. 34. - Nach einer Zeit als Lecturer und Reader wurde er 1951 Professor.

52 Otto Kahn-Freund, Labour Law and Politics in the Weimar Republic, Oxford 1981; ders. (wie Anm. 20); vgl. auch Gaetano Vardaro, Otto Kahn-Freund e l'emigrazione dei giuslavoristi weimariani, in: Politica del diritto 1982, 77-100. - Siehe auch das Gespräch mit Wolfgang Luthardt Anm. 20. - Vgl. auch Wolfgang Däubler, in: Streitbare Juristen (wie Anm. 11), 380-389. 
logisch Relevantes: eine Einführung zur Neuausgabe von Karl Renner „Die Rechtsinstitute des Privatrechts und ihre soziale Funktion“ ${ }^{53}$ und „Labour and the Law“. 54

Eine Sonderstellung nimmt allerdings Franz Neumann ein. ${ }^{55}$ Neumann pendelte zwischen den USA und Westdeutschland/Berlin. Hier wurde er aktiv bei der Beförderung der Politikwissenschaft, vor allem in Berlin an der 1949 wieder gegründeten Hochschule für Politik, an der er ja in der Weimarer Zeit (1928-1933) tätig war. 1953 verlieh ihm die FU Berlin den Dr. phil. h.c. (nicht den Dr. iur. h.c.). Man sollte allerdings auch eine weitere interessante Konstellation beachten: In seinem Todesjahr 1954 erschien der erste Band eines Handbuchs über die Grundrechte - von ihm gemeinsam herausgegeben mit niemand anderen als Hans Carl Nipperdey, einem führenden Arbeitsrechtler vor und nach 1933, ${ }^{56}$ und Ulrich Scheuner, seit 1933 ordentlicher Profes-

53 Englisches Original 1949; deutsche Übersetzung Stuttgart 1965, 1-44, und auch die Anmerkungen sind von Kahn-Freund, 207-286. - Die Arbeit von Karl Renner (1870-1950) - SPÖMitglied und nach 1945 Bundespräsident der Republik Österreich - zählt zu den Klassikern einer marxistischen Rechtstheorie: 1904 noch unter dem Pseudonym Josef Karner, „Die soziale Funktion der Rechtsinstitute besonders des Eigentums“, dann 1929 unter seinem richtigen Namen, aber mit leicht verändertem Titel erschienen: „Die Rechtsinstitute des Privatrechts und ihre soziale Funktion“. Renner war übrigens auch in den Jahren 1932/33 Mitherausgeber von „Die Justiz“ (Anm. 10).

54 Englisches Original 1972, deutsche Übersetzung: Arbeit und Recht, Frankfurt a.M. 1979.

55 Ein kleiner Exkurs zu einer interessanten Konstellation: 1949 hatte Neumann noch eine englische Übersetzung von Montesquieus „Geist der Gesetze“ herausgebracht, vgl. Montesquieu, The Spirit of the Laws, ed. Franz Neumann, transl. Thomas Nugent, New York 1949. - Die deutsche Übersetzung der Einleitung erschien in: Herbert Marcuse (Hg.), Franz Neumann. Demokratischer und autoritärer Staat. Studien zur politischen Theorie, Frankfurt a.M. 1967, 142-194. Ich erwähne das, weil zur selben Zeit in Deutschland Ernst Forsthoff an einer deutschen Übersetzung von Montesquieus Hauptwerk saß - wohl sein rechtsstaatliches Purgatorium -, just zu einer Zeit (1949), als er wieder seinen Lehrstuhl in Heidelberg erhielt. Zur Datierung der Rückkehr nach Heidelberg (1949) siehe Claus-Dietrich Wieland, Personelle Kontinuitäten in der Staatsrechtslehre, in: Restauration im Recht. Jahrbuch für Sozialökonomie und Gesellschaftstheorie, Opladen 1988, 129-154, 143. - Forsthoffs Übersetzung von Montesquieu erschien dann in Tübingen 1951.

56 Hans Carl Nipperdey (1895-1968), Privatdozent Jena 1920; außerordentlicher Professor Jena 1924; ordentlicher Professor Köln 1925. Bereits in Weimar gab er ein dreibändiges Werk über „Die Grundrechte und Grundpflichten der Reichsverfassung“ heraus (Berlin 1929/30). Im Nationalsozialismus profilierte er sich als Arbeitsrechtler, u.a. als Mitkommentator des Gesetzes zur Ordnung der Arbeit; erster Präsident des Bundesarbeitsgerichts 1954-1963. Man beachte auch die Liaison mit Staatssekretär Freisler bei der Herausgabe der Festschrift für Justus Wilhelm Hedemann zum sechzigsten Geburtstag, Berlin 1938. Nur knapp erwähnt wird die Zusammenarbeit von Neumann und Nipperdey bei Thorsten Hollstein, Die Verfassung als „Allgemeiner Teil““. Privatrechtsmethode und Privatrechtskonzeption bei Hans Carl Nipperdey (= Beiträge zur Rechtsgeschichte des 20. Jahrhunderts 51), Tübingen 2008, $115 \mathrm{f}$. Danach hatte sich Neumann in einem Brief vom 5. Oktober 1950 an Nipperdey gewandt mit der Anfrage und Anregung der Herausgabe eines ähnlichen Sammelwerkes wie „Die Grundrechte und Grundpflichten der Reichsverfassung“, das Nipperdey 1929/30 herausgegeben und Neumann 1930 rezensiert hatte, vgl. ebd., 38. 
sor für öffentliches Recht in Jena. ${ }^{57}$ Da möchte man gerne Näheres über dieses Trio erfahren ... 58

Die Überlebenden der Sinzheimer-Gruppe und Kirchheimer blieben nach 1945 persönlich und literarisch verbunden: Fraenkel hielt die Gedenkrede auf Franz Neumann; ${ }^{59}$ Kirchheimer schrieb eine Einführung zu der von Thilo Ramm besorgten Neuherausgabe der „Chronik“ der „Justiz“ mit den Beiträgen von Sinzheimer und Fraenkel; ${ }^{60}$ Kahn-Freund gab gemeinsam mit Thilo Ramm die „Gesammelten Aufsätze und Reden“ zu Arbeitsrecht und Rechtssoziologie von Hugo Sinzheimer heraus und versah sie mit einer Einleitung. ${ }^{61}$

Lebendig geblieben in den theoretischen Debatten ist wohl vor allem Franz Neumann, zu dem sich in der politikwissenschaftlichen Diskussion eine breite Anschlussliteratur findet. ${ }^{62}$

Die Schriften unserer Protagonisten erlebten seit den 60er Jahren Neuauflagen. Es beginnt 1962 mit der Herausgabe von Arbeiten von Hermann Kantorowicz über „Rechtswissenschaft und Soziologie“. Hier geht es anscheinend noch um Interdiszipli-

$57 \mathrm{Zu}$ Scheuner (1903-1981) vgl. Wieland (wie Anm. 55), 146: 1930 Privatdozent Berlin, 1933 ordentlicher Professor Jena, 1940 Göttingen, 1941 Straßburg; NSDAP, SA-Mitglied; 1948 Lehrbeauftragter an der Universität Bonn und der TH Stuttgart; 1950-72 ordentlicher Professor Bonn. - Nipperdey und Scheuner werden übrigens auch im Behemoth zitiert, vgl. Ausgabe 1944: 513 Fn. 14 (Nipperdey), 493 Fn. 76 (Scheuner). - Siehe dann auch deren beider Nachruf auf Neumann in: JZ 10 (1955), $61 \mathrm{f}$.

58 Zuerst erschien Franz Neumann †/Hans Carl Nipperdey/Ulrich Scheuner (Hg.), Die Grundrechte. Handbuch der Theorie und Praxis der Grundrechte, Bd. II, Berlin 1954, 2. unveränderte Aufl. 1968. In den folgenden Teilbänden tauchen als Hg. auf: Bettermann, Nipperdey, Scheuner (III/1 [1958], III/2 [1959], IV/1 [1960]), dann nur noch Bettermann und Nipperdey (IV/2 [1962]); im zuletzt erschienenen ersten Band (1966/67) mit den internationalen Beiträgen, die Neumann eingeworben hatte, sind als Hg. angeführt: Bettermann, Neumann $\dagger$, Nipperdey. Im ersten Teilband dieses Bd. I findet sich die Widmung „Dem Anreger dieses Bandes Franz L. Neumann“. - Laut Vorwort im zuerst erschienenen Bd. II, 1. Teilband, verfasst von Nipperdey und Scheuner, war Neumann „vor 1933 als angesehner Rechtsanwalt in Berlin und Dozent an der Akademie der Arbeit in Frankfurt und dann an der Hochschule für Politik in Berlin tätig ..." - Dieser Zusammenhang zwischen Neumann und Nipperdey ist Rainer Erd und Alfons Söllner in ihrem Gespräch entgangen, die darüber reflektieren, dass nicht Neumann, sondern Nipperdey mit Unterstützung der Gewerkschaften zum ersten Präsidenten des Bundesarbeitsgerichts ernannt wurde, vgl. in: Alfons Söllner, Neumann zur Einführung, Hannover 1982, 97.

59 Gedenkrede auf Franz L. Neumann (1954) in: Fraenkel (wie Anm. 43).

60 Thilo Ramm (Hg.), Die Justiz in der Weimarer Republik. Eine Chronik, Neuwied u.a. 1968.

61 Wie Anm. 9.

62 Joachim Perels (Hg.), Recht, Demokratie und Kapitalismus. Aktualität und Probleme der Theorie Franz L. Neumanns, Baden-Baden 1984; Rainer Erd (Hg.), Reform und Resignation. Gespräche über Franz L. Neumann, Frankfurt a.M. 1985; Joachim Rückert, Franz Leopold Neumann (1900-1954) - ein Jurist mit Prinzipien, in: Marcus Lutter u.a. (Hg.), Der Einfluß deutscher Emigranten auf die Rechtsentwicklung in den USA und in Deutschland. Vorträge und Referate des Bonner Symposions im September 1991, Tübingen 1993, 437-474; Peter Intelmann, Franz L. Neumann. Chancen und Dilemma des politischen Reformismus (= Nomos-Universitätsschriften - Politik 1996), Baden-Baden 1996; Mattias Iser/ David Strecker (Hg.), Kritische Theorie der Politik. Franz L. Neumann - eine Bilanz, BadenBaden 2002. 
narität, noch nicht um die Rekonstituierung der Rechtssoziologie als einer eigenen Disziplin. Das geschieht wohl erst seit $1964 .^{63}$

Das konjunkturelle Wiedererstarken der Rechtssoziologie seit 1964 speist sich aus zwei Quellen: einmal aus der Wiederentdeckung der links-kritischen Tradition der Autoren aus der Weimarer Zeit und des Exils, zum anderen aus der Neubelebung des Traditionsstranges, der von Eugen Ehrlich über Arthur Nußbaum zu Ernst E. Hirsch und Manfred Rehbinder reicht. In der Folgezeit erlebte die Rechtssoziologie in der Bundesrepublik eine neue Blüte vor allem als eine empirisch orientierte Disziplin mit den Untersuchungsfeldern in den Bereichen Justizforschung, Richtersoziologie, Zugang zum Recht, Alternativen zum Recht und der Rechtswirkungs- oder Effektivitätsforschung. Durch die zahllosen Beiträge Niklas Luhmanns zur Rechtssoziologie ergab sich eine Spaltung der Disziplin in einen eher empirischen und einen eher großtheoretischen Bereich.

63 Neupublikationen der alten Schriften in chronologischer Folge:

1962: Thomas Würtenberger (Hg.), Hermann Kantorowicz. Rechtswissenschaft und Soziologie. Ausgewählte Schriften zur Wissenschaftslehre, Karlsruhe.

1964: Theodor Geiger, Vorstudien zu einer Soziologie des Rechts, Neuwied/Berlin, eingeleitet von Paul Trappe (Neuauflage hg. von Manfred Rehbinder, Berlin 1987); Otto Kirchheimer, Politik und Verfassung, Frankfurt a.M.

1966: Thilo Ramm (Hg.), Arbeitsrecht und Politik. Quellentexte 1918-1933, Neuwied/Berlin [Arbeiten von Fraenkel, Neumann und Kahn-Freund].

1967: Otto Kirchheimer, Politische Herrschaft. Fünf Beiträge zur Lehre vom Staat, Frankfurt a.M.; Herbert Marcuse (Hg.), Franz Neumann. Demokratischer und autoritärer Staat. Studien zur politischen Theorie, eingeleitet von Helge Pross, Frankfurt a.M.

1968: Thilo Ramm (Hg.), Die Justiz in der Weimarer Republik, Neuwied/Berlin („Chronik“ der „Justiz“ mit den Beiträgen von Sinzheimer und Fraenkel, Einführung von Kirchheimer); Ernst Fraenkel, Zur Soziologie der Klassenjustiz und Aufsätze zur Verfassungskrise 1931-1932, mit einem Vorwort zum Neudruck, Darmstadt; Manfred Weiß (Hg.), Ludwig Bendix. Zur Psychologie der Urteilstätigkeit des Berufsrichters und andere Schriften, Neuwied u.a.; Manfred Rehbinder(Hg.), Arthur Nußbaum. Die Rechtstatsachenforschung, Berlin. 1972: Otto Kirchheimer, Funktionen des Staates und der Verfassung. 10 Analysen, Frankfurt a.M.

1974: Ernst Fraenkel, Der Doppelstaat, Frankfurt a.M. u.a.; Georg Rusche, Otto Kirchheimer, Sozialstruktur und Strafvollzug, Frankfurt a.M. u.a. (weitere Neuauflage vermehrt um einen Anhang mit zwei Aufsätzen von Georg Rusche und um ein Nachwort von Heinz Steinert, ebd. 1981).

1976: Otto Kahn-Freund, Thilo Ramm (Hg.), Hugo Sinzheimer. Arbeitsrecht und Rechtssoziologie. Gesammelte Aufsätze und Reden, 2 Bde., Frankfurt a.M. u.a. (mit einer Einführung von Otto Kahn-Freund, Bd. 1, 1-31); Wolfgang Luthardt (Hg.), Otto Kirchheimer. Von der Weimarer Republik zum Faschismus: Die Auflösung der demokratischen Rechtsordnung, Frankfurt a.M.

1977: Gert Schäfer (Hg.), Franz Neumann. Behemoth. Struktur und Praxis des Nationalsozialismus 1933-1944, Köln u.a.

1978: Alfons Söllner (Hg.), Franz Neumann. Wirtschaft, Staat, Demokratie. Aufsätze 1930-1954, Frankfurt a.M.

1980: Franz Neumann, Die Herrschaft des Gesetzes, Frankfurt a.M., Übersetzung von Alfons Söllner.

1999: Alexander von Brünneck (Hg.), Ernst Fraenkel. Gesammelte Schriften, Bd. 1: Recht und Politik in der Weimarer Republik, Bd. 2: Nationalsozialismus und Widerstand (darin: Der Doppelstaat und Urdoppelstaat), Baden-Baden. 
In den letzten Jahren ist ein Exodus der deutschsprachigen Rechtssoziologie aus der akademischen Landschaft zu verzeichnen. Bekannte Vertreter der Disziplin wurden pensioniert: Blankenburg, Gessner, Lautmann, Raiser, Manfred Rehbinder, Röhl - ohne dass ihre Lehrstühle, wenn sie denn überhaupt erhalten blieben, mit ähnlich stark an der Rechtssoziologie orientierten Vertretern wieder besetzt worden wären. Das von Hirsch und Rehbinder gegründete Institut für Rechtssoziologie und Rechtstatsachenforschung wird vielleicht noch das Jahr 2012 erleben. Nach 1990 ist es in den neuen Bundesländern nicht gelungen, auch nur eine Professur mit rechtssoziologischem Schwerpunkt zu etablieren (in Halle wird noch die Fahne hochgehalten; an der Humboldt-Universität könnte das Bäumchen Rechtssoziologie wieder wachsen). Aus der rechtswissenschaftlichen Ausbildung droht die Rechtssoziologie zu verschwinden. Bei den Soziologen spielt sie ohnedies kaum eine Rolle. Nur wenige juristische Fachbereiche bieten die Rechtssoziologie und andere „Grundlagenfächer“ im Schwerpunktstudium an. Es hat sich auch eine neue Art von Exil ergeben: Rechtssoziologie ist heute eher zu finden in Zürich, Luzern und in der britischen Diaspora. Dass die Rechtssoziologie von Beginn an ihre Konjunkturen hatte, vermag da nur ein wenig zu trösten. 


\begin{tabular}{|c|c|c|c|c|}
\hline 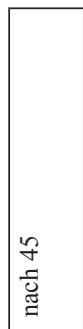 & 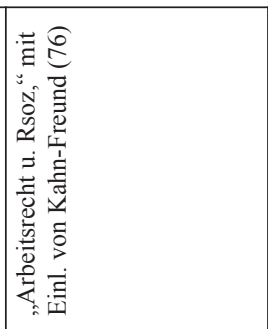 & 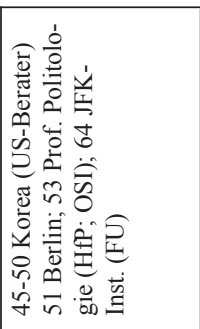 & 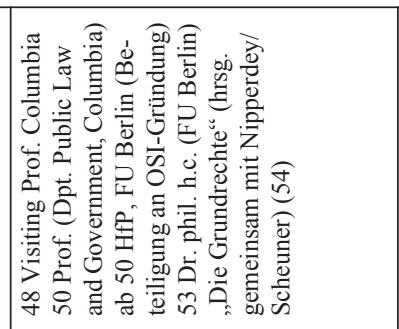 & 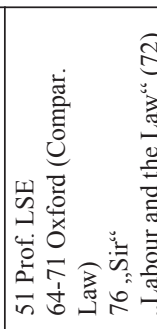 \\
\hline 焉 & 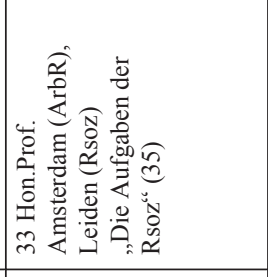 & 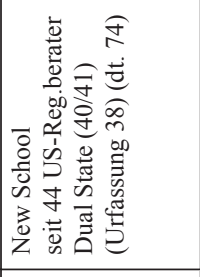 & 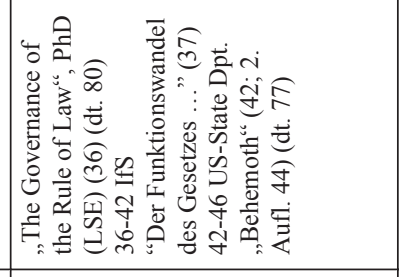 & 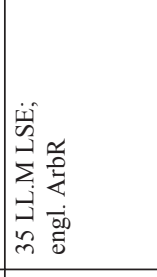 \\
\hline 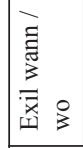 & 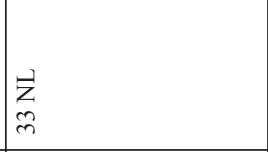 & 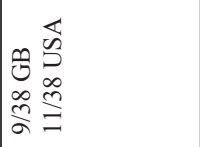 & 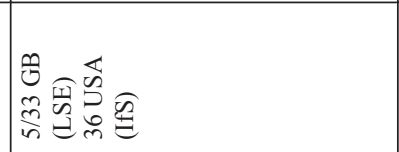 & 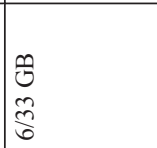 \\
\hline 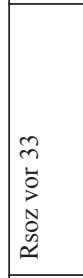 & 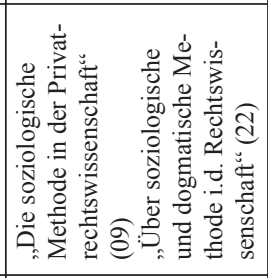 & 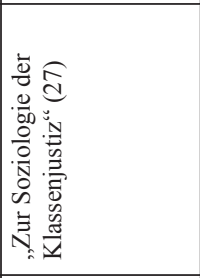 & 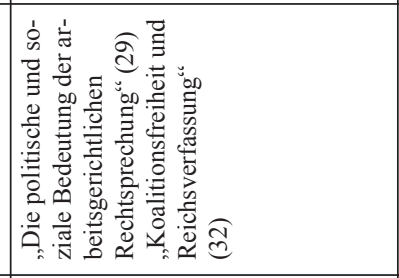 & 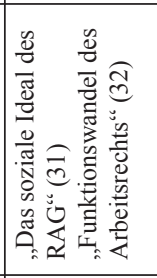 \\
\hline 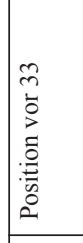 & 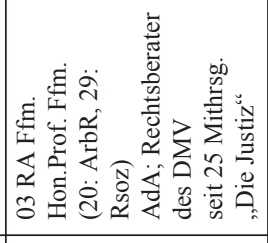 & 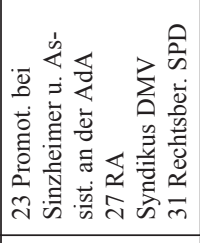 & 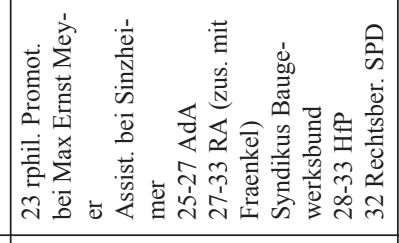 & 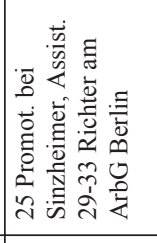 \\
\hline$\stackrel{\varrho}{\Xi}$ & * & * & * & 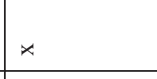 \\
\hline 焉意 & 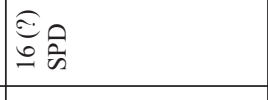 & ते ڤิ & $\begin{array}{l}\hat{\tilde{y}} \\
\text { gे 今े }\end{array}$ & तิ \\
\hline 悹 & 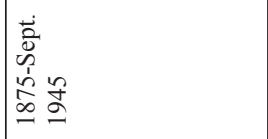 & $\mid \begin{array}{l}n \\
\vdots \\
\dot{\alpha} \\
\infty \\
\infty \\
\infty\end{array}$ & 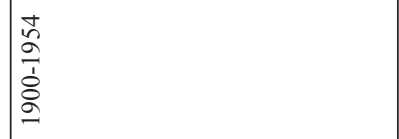 & 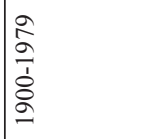 \\
\hline & 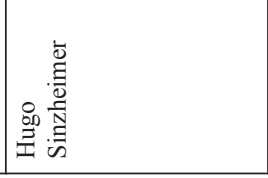 & 总离 & 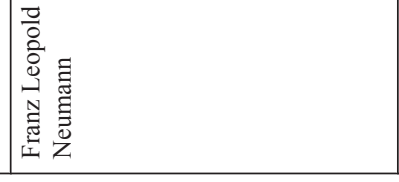 & 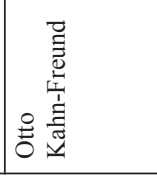 \\
\hline
\end{tabular}




\begin{tabular}{|c|c|c|c|c|}
\hline 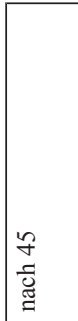 & 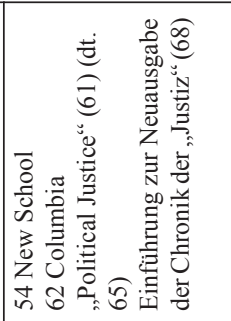 & 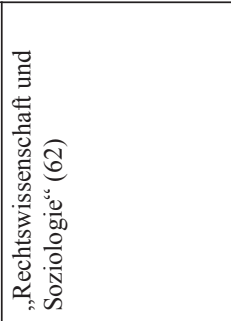 & 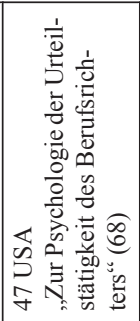 & 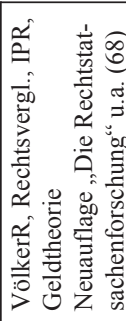 \\
\hline 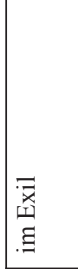 & 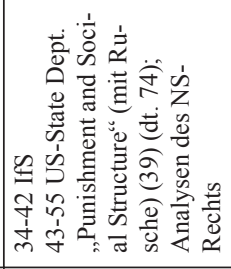 & 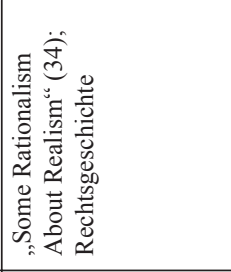 & 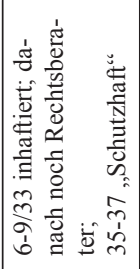 & 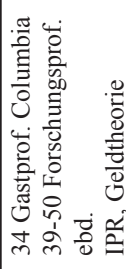 \\
\hline 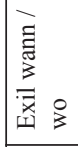 & 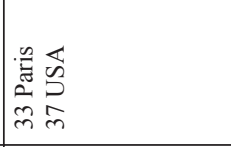 & 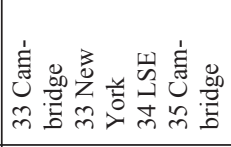 & 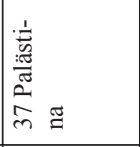 & 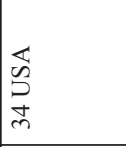 \\
\hline 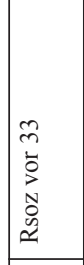 & 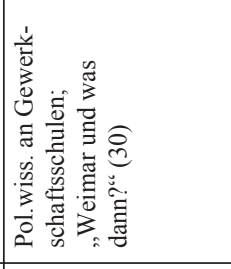 & 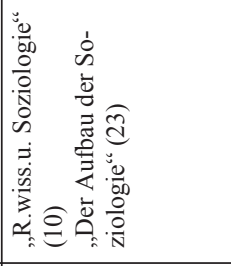 & 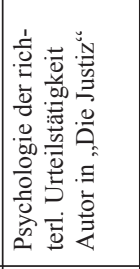 & 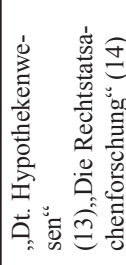 \\
\hline 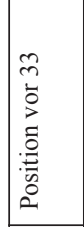 & 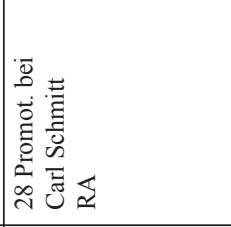 & 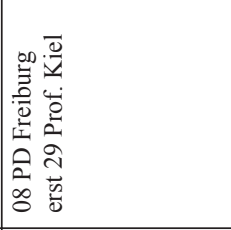 & 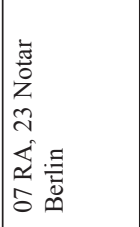 & 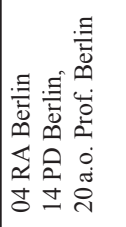 \\
\hline$\stackrel{\Xi}{\Xi}$ & $\star$ & $*$ & $x$ & $\star$ \\
\hline 总言 & $\hat{\tilde{\omega}}$ & 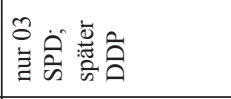 & & \\
\hline 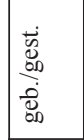 & 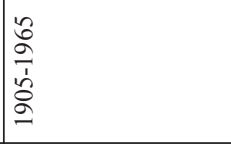 & $\begin{array}{l}\frac{1}{9} \\
\frac{1}{1} \\
\frac{1}{\infty} \\
\frac{1}{1}\end{array}$ & 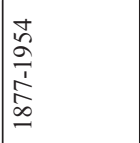 & 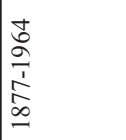 \\
\hline & ) & 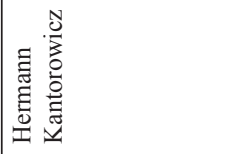 & 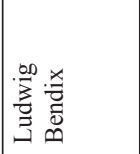 & 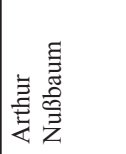 \\
\hline
\end{tabular}




\begin{tabular}{|c|c|c|c|}
\hline 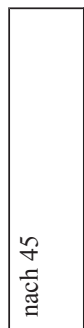 & 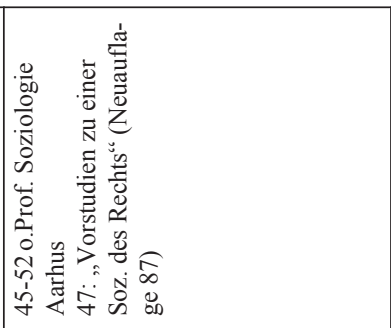 & 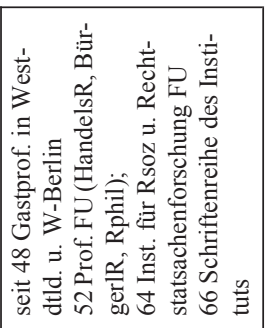 & 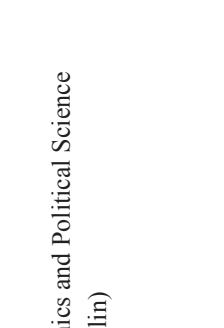 \\
\hline $\begin{array}{l}\text { 离 } \\
\text { 吉 }\end{array}$ & 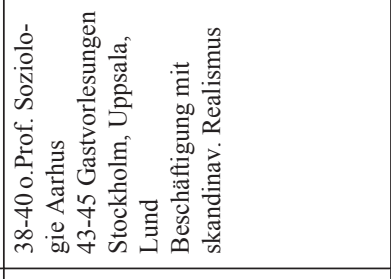 & 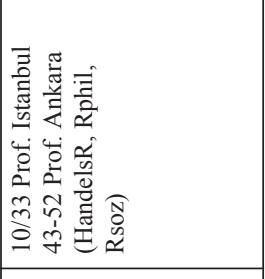 & 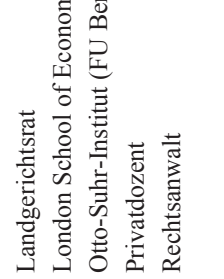 \\
\hline 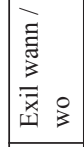 & 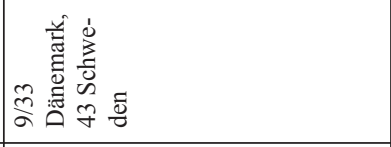 & 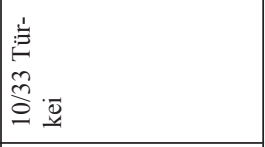 & 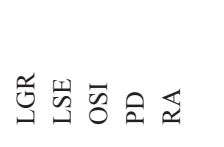 \\
\hline 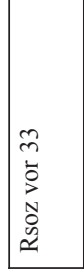 & 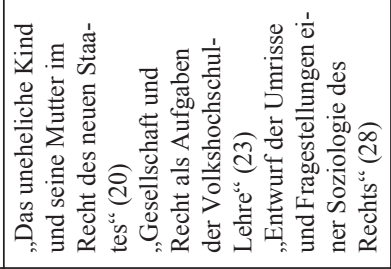 & . & 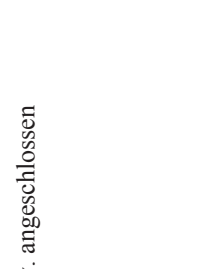 \\
\hline 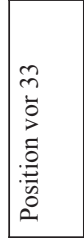 & 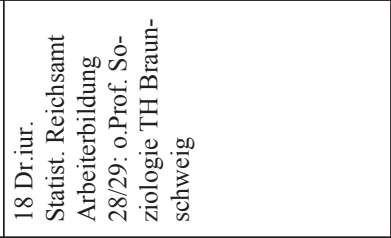 & 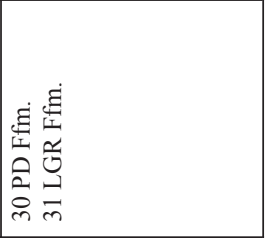 & 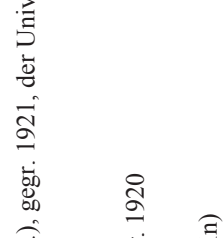 \\
\hline$\frac{g}{\Xi}$ & . & $x$ & 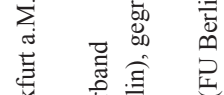 \\
\hline 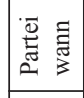 & 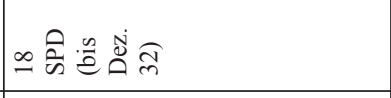 & & 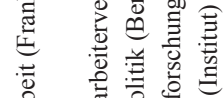 \\
\hline 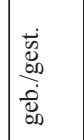 & $\begin{array}{l}\tilde{2} \\
\stackrel{2}{1} \\
\dot{a} \\
\stackrel{\infty}{-}\end{array}$ & 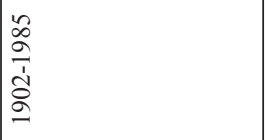 & 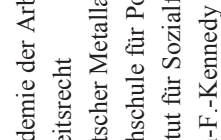 \\
\hline & 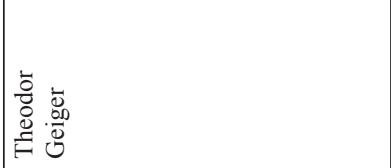 & 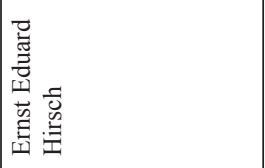 & 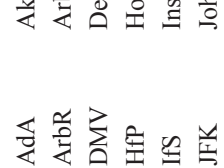 \\
\hline
\end{tabular}

